\title{
Spin-1 gravitational waves and their natural sources
}

\author{
F. Canfora and G. Vilasi \\ Dipartimento di Fisica "E.R.Caianiello", Università di Salerno. \\ Istituto Nazionale di Fisica Nucleare, GC di Salerno, Italy.
}

November 6, 2018

\begin{abstract}
Non-vacuum exact gravitational waves invariant for a non Abelian twodimensional Lie algebra generated by two Killing fields whose commutator is of light type, are described. The polarization of these waves, already known from previous works, is related to their natural sources consisting of cosmic strings and $\gamma$-ray bursts. Non vacuum exact gravitational waves admitting only one Killing field of light type are also discussed.

PACS numbers: 04.20.-q, 04.20.Gz, 04.20.Jb .
\end{abstract}

\section{Introduction}

Because of experimental efforts devoted to the detection of gravitational waves, there is a growing theoretical interest in describing the emission of detectable gravitational waves from astrophysical sources. All experimental devices, laser interferometers or resonant antennas, need very precise theoretical predictions, and usually they are calibrated according to results of linearized theory. In fact, it is commonly believed that, since the sources are very far from the earth, the wave's amplitude is so small at the earth surface that the use of the linearized theory is lawful. However, in some cases this assumption is misleading. Indeed, as shown by Christodoulou [3] the nonlinearity of the Einstein equations manifests itself in a permanent displacement of the test masses of a laser interferometer which, in many physically interesting cases (e.g. for a binary coalescence), cannot be neglected. Moreover, the nonlinearity of the Einstein theory is one of its most characteristic feature, and it is likely that some crucial properties of the gravitational field are encoded in the nonlinear terms. Thus, a detailed analysis of exact gravitational waves, besides to help the calibration of the experimental devices, could disclose new phenomena hidden by the approximations. 
In recent papers exact solutions [14] of vacuum Einstein field equations, representing spin-1 gravitational waves $[4,5]$, have been discussed. They are described by the following metrics:

$$
g=2 f\left(d x^{1} d x^{1}+d x^{2} d x^{2}\right)+\mu\left[\left(w\left(x^{1}, x^{2}\right)-2 x^{4}\right) d x^{3} d x^{3}+2 d x^{3} d x^{4}\right]
$$

where, $\mu=D \Phi+B ; D, B \in \mathcal{R}, \Phi$ is a non constant harmonic function of $x^{1}$ and $x^{2}, f=(\nabla \Phi)^{2} \sqrt{|\mu|} / \mu$, and $w\left(x^{1}, x^{2}\right)$ is a non constant ${ }^{1}$ solution of the $\mu$-deformed Laplace equation:

$$
\Delta w+\left(\partial_{x^{1}} \ln |\mu|\right) \partial_{x^{1}} w+\left(\partial_{x^{2}} \ln |\mu|\right) \partial_{x^{2}} w=0,
$$

which, when $\mu$ is a constant function, reduces to the Laplace equation.

These metrics are intrinsically characterized [14] by the following properties:

- They are invariant for the non Abelian two-dimensional Lie algebra generated by the Killing vector fields $X$ and $Y$ such that:

$$
[X, Y]=Y,
$$

with $Y$ of light-type, i.e., $g(Y, Y)=0$.

- The distribution $\mathcal{D}^{\perp}$, orthogonal to the distribution $\mathcal{D}$ generated by $X$ and $Y$, is integrable and transversal to $\mathcal{D}$.

In the following, an integral (two-dimensional) submanifold $\mathcal{S}$ of $\mathcal{D}$ will be called a Killing leaf and an integral (two-dimensional) submanifold $\mathcal{W}$ of $\mathcal{D}^{\perp}$ will be called a orthogonal leaf.

In [14] it is shown that these Einstein manifolds have a have a natural fibered structure with the Killing leaves as fibers and a basis diffeomorphic to the orthogonal laves.

In the coordinates $\left(x^{\mu}\right)$, which are adapted [14] to the Killing fields, the Killing vector fields $X$ and $Y$ read

$$
X=\frac{\partial}{\partial x^{3}}, \quad Y=\exp \left(x^{3}\right) \frac{\partial}{\partial x^{4}} .
$$

In the particular case $f=1 / 2$ and $\mu=1$, the above metrics are locally diffeomorphic to a subclass of the vacuum Peres solutions [8] corresponding to a special choice of the harmonic function parameterising that metrics.

This is easily seen by introducing, for $u \neq 0$, new coordinates $(x, y, u, v)$ defined by

$$
x^{1}=x, \quad x^{2}=y, \quad x^{3}=\ln |u|, \quad x^{4}=u v,
$$

\footnotetext{
${ }^{1}$ When $w$ is constant, metrics (1) are static.
} 
in which metrics (1) read

$$
g=2 f(x, y)\left[d x^{2}+d y^{2}\right]+\mu(x, y)\left[2 d u d v+\frac{w}{u^{2}} d u^{2}\right] .
$$

A crucial problem in the theory of the gravitational waves is to characterise realistic sources able to generate waves so strong to be detected by the experimental devices. Thus, the aim of this paper is to find physically interesting sources for the above metrics and to relate the physical properties of such gravitational waves (in particular, the asymptotic flatness, the wave-like character and the polarization) to the sources. The plan of the paper is the following: in the first section, we will solve the non-vacuum Einstein equations for metrics (1) with a dust source describing a null electromagnetic wave. In the second section we will discuss the role of the asymptotic flatness and the related constraints on the metric components and on the source. In this section we will also discuss cosmic strings as a possible source. Then we will briefly explain why the standard textbook analysis of gravitational waves does not apply to this case. In the third section we will show that such gravitational fields indeed represent gravitational waves by using two well known covariant criteria: the Zel'manov's and the Pirani's criteria. In the fourth section we will see that such waves are spin-1 objects. In the last section we will give a hopefully vague discussion on the possibility to detect such non standard gravitational waves.

\section{The dust and cosmic string sources}

The simplest source for metrics (1) is dust with density $\rho$ and velocity $U^{\mu}$ and, then, characterized by an energy-momentum tensor $T_{\mu \nu}=\rho U_{\mu} U_{\nu}$. When $U^{\mu}$ is a light-like vector field, this kind of energy-momentum tensor can describe the energy and momentum of null electromagnetic waves, i.e., electromagnetic fields whose scalars, $F^{\mu \nu} F_{\mu \nu}$ and $\epsilon^{\alpha \beta \mu \nu} F_{\alpha \beta} F_{\mu \nu}$, both vanish. In this way it could be possible to describe the gravitational effects (in particular the emission of gravitational waves) of a very interesting astrophysical phenomenon, the $\gamma$-ray bursts (GRBs): emission of ultra-high energetic $\gamma$-rays, whose origin is still to be fully understood.

Moreover, with this source one can also preserve the symmetries $[14,4,5]$ of the vacuum solution.

Being the time coordinate in the Killing leaves, the dust cannot move orthogonally to them and it will be chosen to move parallel to the light-like Killing field $Y$, i.e., with velocity $U^{\mu}=\delta^{\mu 4}$.

Thus, the metric will be taken in the form

$$
\begin{aligned}
g= & 2 F\left(x^{1}, x^{2}\right)\left(\left(d x^{1}\right)^{2}+\left(d x^{2}\right)^{2}\right) \\
& +M\left(x^{1}, x^{2}\right)\left[W\left(x^{1}, x^{2}, x^{4}\right)\left(d x^{3}\right)^{2}+2 d x^{3} d x^{4}\right]
\end{aligned}
$$


with $F, M$ and $W$ to be determined by the non vacuum Einstein equations with the following energy-momentum tensor

$$
T_{\mu \nu}=M^{2} \rho \delta_{\mu 3} \delta_{\nu 3}
$$

Looking at the explicit expression of the Einstein tensor $G_{\mu \nu}$ it turns out that

$$
G_{33}=W G_{34}+\frac{1}{2 F}(M \Delta W+\nabla M \cdot \nabla W),
$$

where

$$
\nabla=\left(\frac{\partial}{\partial x^{1}}, \frac{\partial}{\partial x^{2}}\right), \quad \nabla M \cdot \nabla W=\frac{\partial M}{\partial x^{1}} \frac{\partial W}{\partial x^{1}}+\frac{\partial M}{\partial x^{2}} \frac{\partial W}{\partial x^{2}} .
$$

Then, since $T_{\mu \nu}=0$ for $\mu, \nu \neq 3$, we can fulfil the non vacuum field equations, while keeping the vacuum Killing fields, if and only if $F=f$ and $M=\mu$, i.e. $F$ and $M$ coincide with their vacuum expression [14], and $W$ has the form $W\left(x^{1}, x^{2}, x^{4}\right) \equiv w\left(x^{1}, x^{2}\right)-2 x^{4}, w$ being a solution of the $\mu$-deformed Poisson equation

$$
\mu \Delta w+\nabla \mu \cdot \nabla w=2 f \mu^{2} \rho,
$$

where, to save writing, beyond $c=1$, it has been taken $8 \pi G=1, G$ being the Newton gravitational constant.

The comparison of Eqs. (2) and (6) shows that the introduction of the matter source (5) induces only a simple and natural modification in the field equations. The reason behind this simplicity is that the above source, besides being physically interesting, does not break the geometrical structures which allows [14] to classify completely Ricci-flat metrics of the form (1). In particular, in this case it is still true that the 2-dimensional distributions orthogonal to the surface spanned by the Killing fields is integrable since the energy-momentum tensor has no components orthogonal to the Killing leaves. Thus, the bundle structure [14] of the vacuum space-time is not destroyed by the source.

\section{Asymptotic flatness}

From the physical point of view, it is important to characterise, among the metrics (1), those which are spatially asymptotically flat. The vacuum case, where (for $\mu=1$ ) $x^{1}$ and $x^{2}$ are harmonic coordinates, suggests to call (spatially) asymptotically flat a metric approaching, for $\left(x^{1}\right)^{2}+\left(x^{2}\right)^{2} \rightarrow \infty$, the Minkowski metric.

This intuitive definition of asymptotic flatness allows to obtain qualitative results by using the standard theory of partial differential equations.

In terms of the functions $f, \mu$ and $w$, the asymptotic flatness condition reads:

$$
\begin{aligned}
\left(x^{1}\right)^{2}+\left(x^{2}\right)^{2} & \rightarrow \infty \Rightarrow \\
f & \rightarrow \text { const }, \quad \mu \rightarrow \text { const }, \quad w \rightarrow c_{1} x^{1}+c_{2} x^{2}+c_{3},
\end{aligned}
$$


where $c_{1}, c_{2}$ and $c_{3}$ are arbitrary constants and the behaviour of $w$ can be easily recognized by looking at the Riemann tensor.

It is easy to see that, in order for metrics (1) to be spatially asymptotically flat, necessarily $\mu$ must be constant. Indeed, being $f$ and $\mu$ related by

$$
f=\frac{\sqrt{|\mu|}}{\mu}(\nabla \Phi)^{2}, \quad \mu=A \Phi+B, \quad \Delta \Phi=0,
$$

the condition $\mu \rightarrow$ const implies $A=0$ or $\Phi \rightarrow$ const. However, the case $\Phi \rightarrow$ const has to be excluded because of the nondegeneracy of the metric.

For $\mu=1$, the equation for $w$ reduces to the two-dimensional Poisson equation

$$
\Delta w=\rho .
$$

It is well known that, if $\rho$ goes to zero fast enough, it is possible to find non trivial everywhere regular solutions $w$ tending to a constant value ${ }^{2}$.

The function $f$ satisfies the equation

$$
f \Delta f-(\nabla f)^{2}=0
$$

and this implies that the function

$$
\psi=\ln |f|
$$

is harmonic. Therefore, to have $f$ asymptotically constant, the function $\psi$ must be asymptotically constant. But this is impossible unless $\psi=$ const. Thus, in order to have everywhere regular spatially asymptotically flat solutions, $f$ and $\mu$ must be constant functions and the fluid density $\rho$ must tend to zero fast enough.

However, if we admit $\delta$-like singularities in the $\left(x^{1}, x^{2}\right)$ plane (i.e., stringlike singularities, by taking into account the third spatial dimension), spatially asymptotically flat vacuum solutions with $f \neq$ const and $w \neq$ const can exist. It is worth noting that, in this limiting case in which $\rho\left(x^{1}, x^{2}\right) \rightarrow \delta\left(x^{1}, x^{2}\right)$, the energy-momentum tensor becomes the one usually employed to describe the gravitational effects of topological defects known as cosmic strings [15]. This kind of extended objects are predicted in some particles physics cosmological models with phase transitions. Moreover, cosmic strings could have an important role in the description of two very interesting, but not yet fully understood, astrophysical phenomena: the GRBs and the ultra high energy cosmic rays, i.e. cosmic rays with energy $E \gtrsim 10^{11} \mathrm{GeV}$, (for a review, see, for example, [13]). It is important to stress here that cosmic strings are not singular objects and should be described by a regular energy-momentum tensor, but the above widely used scheme is useful for practical computation.

\footnotetext{
${ }^{2}$ In the vacuum case this is not possible, unless $w=$ const, because the Laplace equation has not solution of this kind, as can be easily checked, for example, by using the maximum principle.
} 
Thus, from the phenomenological point of view, it is very intriguing that these kind of (wave-like, as we will show later on) gravitational fields are naturally coupled to cosmic strings. Eventually, unlike the electromagnetic (or the standard gravitational) waves that can be everywhere regular even without sources, these gravitational fields, to be globally nonsingular, have to be coupled with matter sources. To be more precise, the reason why in the standard textbook analysis of the polarization spin-1 gravitational waves always appear as pure gauges is that one usually considers only square-integrable perturbations (in fact the Fourier analysis is needed to kill such spin-1 components) to the Minkowski metric. However it is easy to verify that, if one considers non square-integrable perturbations, the spin -1 components cannot be gauged away anymore [4]. In the case we are here considering, the physical components of the gravitational field are not square-integrable and represent (as we will see later on) spin-1 gravitational waves.

\section{Wave-character of the field}

From now on, to have a clearer physical interpretation, only the case $f=1 / 2$ and $\mu=1$ will be considered. In fact, in this case the metric (1), besides being an exact solutions of the non vacuum Einstein equations, is a solution of the linearized Einstein equations on flat background too.

As it has been said, metrics (1) and (3) are locally diffeomorphic. Moreover, for $f=1 / 2$ and $\mu=1$, the metric (3) is a particular case of the Peres metric $[8]$ :

$$
d s^{2}=d x^{2}+d y^{2}+2 d u d v+h(x, y, u) d u^{2}
$$

where $h$ is an arbitrary function of $u$. If $h_{x x}+h_{y y}=0$, the metric (7) is a solution of vacuum Einstein field equation, otherwise, since one still has $g^{\mu \nu} R_{\mu \nu}=0$ and $g^{\mu \nu} R_{\mu \alpha} R_{\beta \nu}=0$, the source can be interpreted as a null electromagnetic field $[7,9]$.

With the chosen energy-momentum tensor, the non-vacuum Einstein equations for the metric (7) reduce to

$$
\Delta h=\Delta \frac{w}{u^{2}}=\frac{\rho}{u^{2}} .
$$

Now, let us briefly analyze the non vacuum Peres metric.

Firstly, it is trivial to show that $(x, y, u, v)$ are harmonic coordinates. Furthermore, because of the Laplacian operator in Eq. (8), the vacuum (i.e. with $\rho=0)$ Peres solution also can be asymptotically flat only if we admit $\delta$-like singularities in the $(x, y)$ plane. In other words, the vacuum Peres solution is naturally coupled to cosmic strings too. Eventually, it is easy to see that, in general, this metric has only one ${ }^{3}$ (null) Killing field: $V=\partial / \partial v$ and that the

\footnotetext{
${ }^{3}$ Of course, if $h=w / u^{2}$, then there exist an additional Killing field.
} 
non vanishing independent components of the Riemann tensor are:

$$
R_{i u j u}=-\partial_{i j}^{2} h, \quad i, j=x, y \text {. }
$$

The wave character and the polarization of these gravitational fields can be analysed in many ways. For example, we could use the Zel'manov criterion [16] to show that these are gravitational waves and the Landau-Lifshitz pseudotensor to find the propagation direction of the waves $[4,5]$. However, the algebraic Pirani criterion is easier to handle since it determines the wave character of the solutions and the propagation direction both at once. Moreover, it has been shown that, in the vacuum case, the two methods agree [5]. To use this criterion the Weyl scalars must be evaluated according to the Petrov-Penrose classification $[10,11]$.

To perform the Petrov-Penrose classification, one has to choose a tetrad basis with two real null vector fields and two real spacelike (or two complex null) vector fields. Then, according to the Pirani's criterion, if the metric belongs to type $\mathbf{N}$ of the Petrov classification, it is a gravitational wave propagating along one of the two real null vector fields. However, the Peres coordinates are not of the above type and it is necessary to introduce new coordinates adapted to the Petrov-Penrose classification.

By performing the following transformation

$$
x \mapsto x, \quad y \mapsto y, \quad u \mapsto u, \quad v \mapsto v+\varphi(x, y, u)
$$

whose Jacobian is equal to one, the metric (7) with the choice $h=\varphi_{, u}$ becomes:

$$
g=d x^{2}+d y^{2}+2 d u d v+2(\varphi, x d x+\varphi, y d y) d u
$$

and has, for generic $\varphi$, the only Killing field $Y=\partial / \partial v$.

Since $\partial_{u}$ and $\partial_{v}$ are null real vector fields and $\partial_{x}$ and $\partial_{y}$ are spacelike real vector fields, the above set of coordinates is the right one to apply for the Pirani's criterion.

Since the only nonvanishing components of the Riemann tensor, corresponding to the metric (9), are

$$
R_{i u j u}=-\partial_{i j}^{2} \partial_{u} \varphi, \quad i, j=x, y,
$$

this gravitational fields belong to Petrov type $\mathbf{N}([2,16])$. Then, according to Pirani's criterion, the metric (9) does indeed represent a gravitational wave propagating along the null vector field $\partial_{u}$. It is worth noting here that, in order to have a spatially asymptotically flat gravitational field, $\partial_{u} \varphi$ has to be a solution of the two-dimensional Poisson equation in approaching a constant at infinity; therefore, $\partial_{u} \varphi, \varphi_{, x}$ and $\varphi, y$ are not square-integrable functions. 


\section{Polarization}

Once the propagation direction has been determined, to compute the polarization we only need to look at the transformation properties of the physical components of the metric (9) under a rotation in the $(x, y)$ plane orthogonal to the propagation direction. By "physical components" we mean the true degrees of freedom of the metric. Since the metric (9) has only two independent components, we have simply to look at the transformation properties of these components. Moreover, Bel's superenergy tensor singles out the same degrees of freedom [5]. It is worth noting here that the metric (9), besides being an exact solution of the Einstein equations, is also a solution of the linearized Einstein equations on flat background:

$$
\begin{aligned}
g & =d x^{2}+d y^{2}+2 d u d v+2\left(\varphi_{, x} d x+\varphi_{, y} d y\right) d u=\eta_{a b}+h_{a b} \\
h_{a b} & =\delta_{a u} \delta_{b i} \varphi_{, i}
\end{aligned}
$$

where $\eta_{a b}$ is the flat metric and $h_{a b}$ can be considered as the "perturbation". Then, the spin may be discussed using the standard textbooks analysis. However, before working out the transformation properties of the physical components of the metric, we should verify that the system of coordinate is harmonic (otherwise, there would be no meaningful notion of polarization). In fact, by construction, the system of coordinates of the metric (9) is harmonic, so that the standard procedure applies. Thus the analysis of the polarization of this gravitational waves runs in exact the same way as in [5]. The result is the same: these are spin-1 gravitational waves because, roughly speaking, the (non squareintegrable) physical components of these waves $h_{u x}=\varphi_{, x}$ and $h_{u y}=\varphi_{, y}$ have only one index in the $(x, y)$ plane orthogonal to the propagation direction $\partial_{u}$. It is worth noting that these results also hold when the function $f$ in Eq. (1) is not constant. The reason behind this non-standard result could be that the spacetime topology is non trivial due to the singularities of the function $\partial_{u} \varphi$ (as can be easily recognized by considering the case in which $\partial_{u} \varphi$ is a solution of the Poisson equation with $\delta$-like sources, or, in other words, when we consider the physical effects of cosmic strings). The gauge transformations are able to kill spin-1 degrees of freedom only locally, but in this case the singularities seem to be a global obstruction.

The possibility of spin-1 gravitational waves has also been guessed, although in a rather different context, in [1] and [6].

\section{$5 \quad$ Possible experimental tests}

The following is a rather vague discussion on the possibility to detect such non standard gravitational waves. The vagueness of the below comments is mainly related to the lacking of experimental data on gravitational waves. Nevertheless, we believe that it is interesting to outline in a very general (vague) way how 
one could recognize the presence of spin-1 gravitational waves in a would be gravitational wave's bursts detected by a laser interferometer. The effects of these waves on neighbouring test particles can be addressed by using the Jacobi geodesic deviation equation which for the metric (9) reads:

$$
\frac{d^{2}}{d \tau^{2}} Z^{i}=-g^{i h} Z^{j} \partial_{j} \partial_{h} \partial_{u} \varphi
$$

where $Z^{\mu}$ is a spacelike vector, with components only in the $(x, y)$ plane, representing the displacement of two close geodetics and $\tau$ is the proper time along the close geodesics.

The above equation shows that one can have either attraction or repulsion according to the choice of $\varphi$, this $\varphi(x, y, u)$ being constrained, outside the matter source, only by the condition to be a harmonic function of $x$ and $y$. For example, the choice $\varphi=\rho(x, y) \sigma(u)$, with $\rho$ a harmonic function ${ }^{4}$ of $x$ and $y$ and $\sigma$ a decreasing function of $u$, will give repulsion. This is not surprising because it is known from QFT that spin-odd bosons generate repulsion between particles of the same charge, the charge, in this case, corresponding to the mass.

Now, the question is: "Can we hope to detect such spin-1 waves?". From the theoretical point of view, it would be important to have an estimate of the flux of spin-1 gravitational waves. However, it is rather difficult to obtain even a rough estimate of the energy-density of all possible sources of such waves. For this reason an approximate expression for $\rho$ is not available yet, and, without $\rho$, it is not easy to estimate the expected flux. From the experimental point of view, as it is well known, there are delicate technical problems to detect gravitational waves from astrophysical sources because of the weakness of the wave amplitudes with respect to experimental noises. Nevertheless, once it will be possible to "observe" gravitational waves, it could be possible to distinguish the spin- 2 and the spin- 1 components. Indeed, suppose to put the detector (such as a laser interferometer or a resonant antenna) in the $(x, y)$ plane orthogonal to the propagation direction of the waves. By definition, if a spin-2 gravitational wave's burst is observed, then the experimental data have to be invariant under a rotation of $\pi$ in the $(x, y)$ plane. This means that the observable effects of the gravitational wave's burst do not change if the detector is rotate of an angle equal to $\pi$. However, if the burst contains also a spin- 1 component, then the above conclusion is not true anymore. In fact, the observable effects of the spin1 component are invariant under a rotation of $2 \pi$ in the $(x, y)$ plane. Therefore, a breaking of the invariance of the experimental data under a rotation of $\pi$ in the $(x, y)$ plane could be interpreted as the effect of spin- 1 gravitational waves and the amount of such a breaking could be used to get an estimate of the flux of such waves. Moreover, since cosmic strings are naturally coupled to spin-1 gravitational waves, if the device is able to detect mainly cosmological gravitational waves, it could be possible, by looking at the experimental data,

${ }^{4}$ The choice $\rho=\ln \sqrt{x^{2}+y^{2}}$ gives an asymptotic flat solution. 
to put further constraints on the presence of cosmic strings. In fact, even a small spin-1 component could be the signature of a non zero density of cosmic strings in the early cosmology.

\section{References}

[1] D. V. Ahluwalia, N. Dadhich, M. Kirchbach, Int. J. Mod. Phys. D (2002)1621.

[2] S. Chandrasekar, The mathematical theory of black holes, (Clarendon Press, Oxford, 1983).

[3] D. Christodoulou, Phys. Rev. Lett. 67, 1486 (1991).

[4] F. Canfora, G. Vilasi, P. Vitale, Phys. Lett. B, 545 (2002)373-378.

[5] F. Canfora, G. Vilasi, P. Vitale, Spin-1 gravitational waves, gr-qc/0212024; to appear in Int.J.Mod. Phys. B.

[6] M. Kirchbach, D. V. Ahluwalia, Phys. Lett. B, 529 (2002) 124-131.

[7] C. W. Misner, J. A. Weeler, Ann. Phys. (N.Y.) 2, 525 (1957)

[8] A. Peres, Phys. Rev. Lett. 3, 571 (1959)

[9] A. Peres, Phys. Rev. 118, 1105 (1960)

[10] A. Z. Petrov, Einstein spaces, (Pergamon Press, New York 1969)

[11] R. Penrose, Ann, of Phys. 10:171, (1960)

[12] N. Rosen, Bull. Res. Coun. Isr. 3, 328 (1954).

[13] M. Sakellariadou, hep-ph/0212365 (2002).

[14] G. Sparano, G. Vilasi and A.M.Vinogradov, Phys. Lett. B 513, 142 (2001); Diff. Geom. Appl. 16, 95 (2002); 17, 1 (2002).

[15] A. Vilenkin, E. P. S. Shellard, Cosmic Strings and Other Topological Defects, (Cambridge University Press, 2000).

[16] V. D. Zakharov, Gravitational waves in Einstein's theory, (Halsted Press, N.Y.1973) 